\title{
MAPS AVAILABLE
}

(Bibliographic guide prepared courtesy of Dr. J. Gravesteijn, with the assistance of the BRGM, France, and the IUGS Commission on Geological Documentation)

\section{FRANCE (Second Part)}

Feuille disponible

Feuille mise en vente au cours du $1^{\text {er }}$ semestre 1981

Feuille mise en vente au cours du $2^{\text {ème }}$ semestre 1981

Feuille prévue pour 1982

Feuille levée, en préparation

Edition photographique de la carte géologique

Feuille disponible à plat seulement

(Les autres feuilles sont disponibles soit à plat, soit pliées)

General legend for indexes.

\section{Systematic Mapping}

Geology

Carte géologique de la France - 1:320 000 - Orléans: BRGM, (See Index Map 1). Sheet 14, METZ and 29, BAYONNE available on photographic edition.

Carte géologique de la France - 1:250 000 - Orléans: BRGM, In progress. This map will replace the existing 1:320 000 map (See Index Map 2).

Carte géologique détaillée de la France - 1:80 000 - Orléans: BRGM, (See Index Map 3). Sheets 17, 71, 88, 106, 119, 144, $146,167,176,179$ bis, 196, 201, 218, 219, 221, 244, 251, $255,256,261,262,263,265$ available on photographic edition.

COUPURES SPÉCIALES

Carte géologique des Pyrénées aे $1 / 250000$ (S.N.P.A.)

Carte gèologique simplifiée

des Alpes du nord à $1 / 250000$

(Coèdition Didier-Richard-B.R.G.M.)

CARTE GÉOLOGIQUE

A la découverte des paysages géologiques de PARIS à AVALLON à $1 / 200000$

A la découverte des paysages géologiques de MARSEILLE à MENTON à $1 / 200000$

\section{A $1 / 320000$}

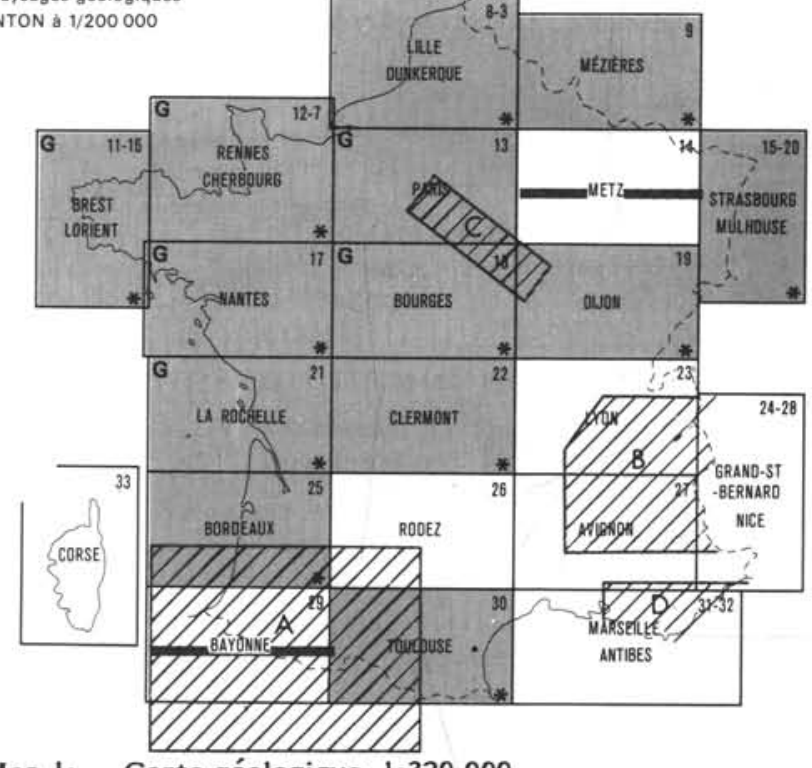

Index Map 1: Carte géologique, 1:320 000

\section{CARTES GÉOLOGIQUES A 1/250 000}

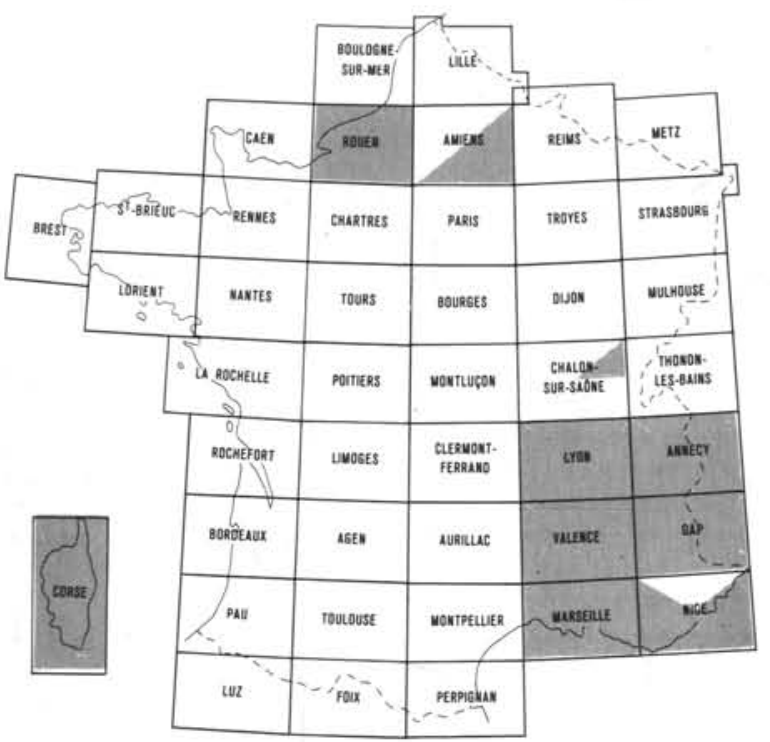

Index Map 2: Carte géologique, 1:250 000

Carte géologique de la France - 1:50 000 - Orléans: BRGM, In progress. This map will replace the existing 1:80 000 map (See Index Map 4).

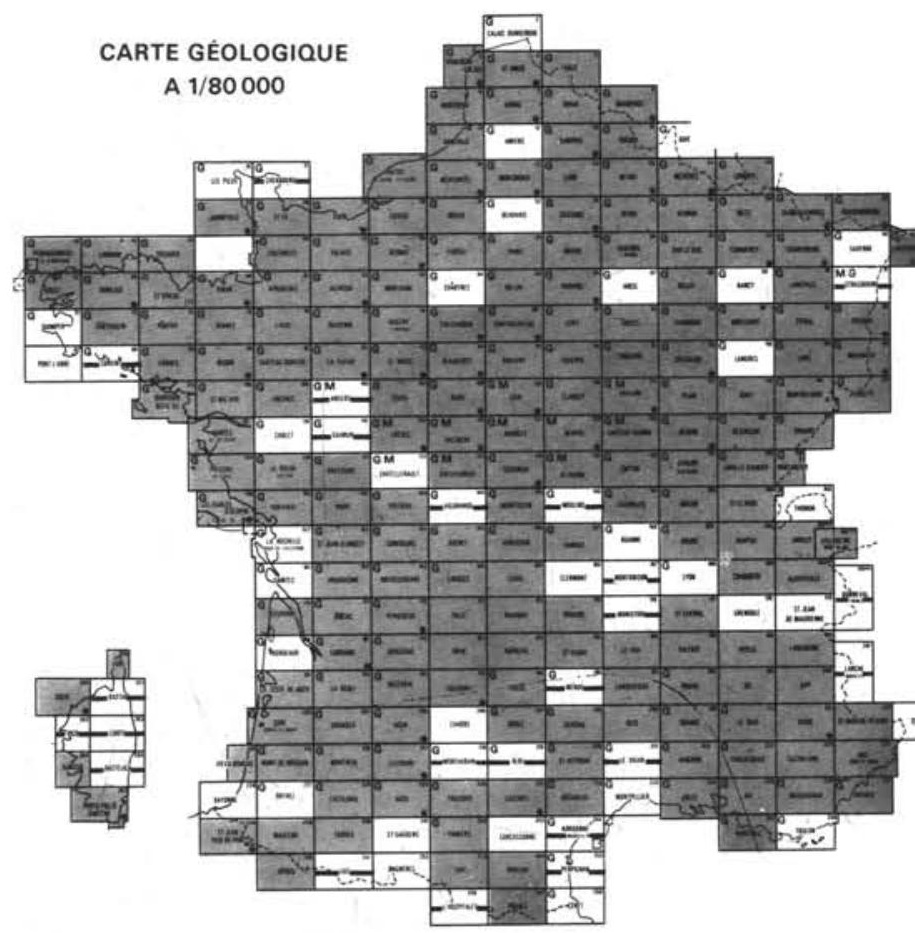

Index Map 3: Carte géologique, 1:80 000 


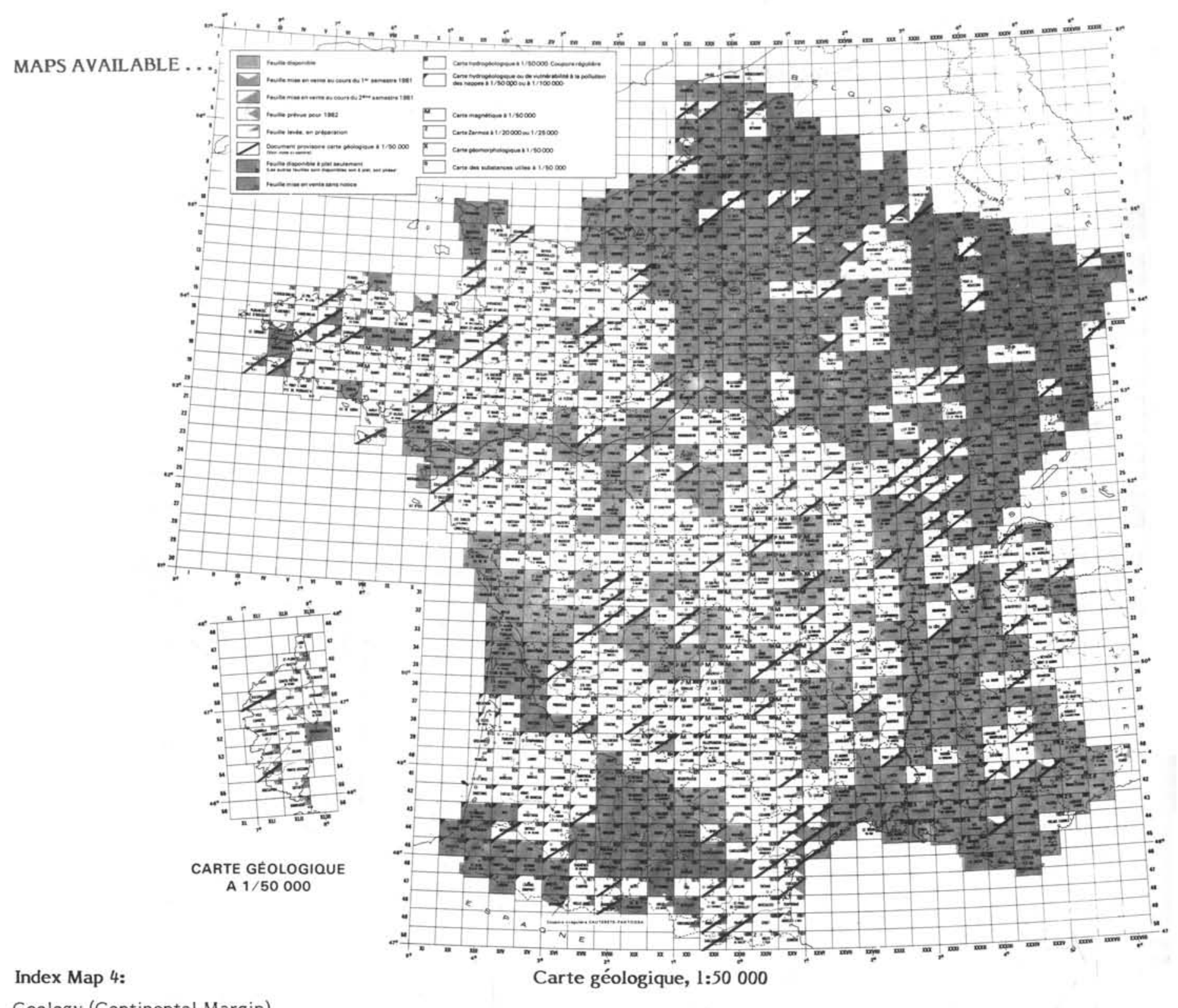

\section{Geology (Continental Margin)}

Carte sédimentologique sous-marine des côtes de France 1:100 000 - Paris: IGN, Sheets published:

Brest, 1968; Bricquebec, 1968; Ile d'Yeu, 1971; Lorient, 1972; Plabennec, 1975; Pont-Croix, 1969; Saint-Nazaire, 1968;), Saint-Vaast-la-Hougue, 1967; (See Index Map 5), Order from: BRGM (see below).

Carte géologique de la marge continentale française: Nature des dépôts meubles superficiels - 1:100 000 - Orléans: BRGM, Sheets published: Argeles-sur-mer, 1972; Marseille, 1972; Saint-Tropez, 1974; (See Index Map 5).

Carte géologique et structurale de la marge continentale française - 1:250 000 - Orléans: BRGM. Sheets published: Boulogne-sur-mer-Rouen, 1971; Caen-Wight, 1971; Ouessant, 1975; Penmarc'h, 1970. Special sheet: Atlas Géologique du Plateau Continental Aquitain, 1976. (See Index Map 6).

\section{Geomorphology}

Carte géomorphologique détaillée de la France - 1:50 000 Paris: CNRS. Sheets published: Les Andelys, Brest, Castellane, Chartres, Courville-sur-Eure, Dreux, Evreux, Grenoble, Narbonne, Nogent-le-Roi, Saint-André-de-l'Eure, Saint-Chely-d'Apcher, Saint-Girons, Saugues, Strasbourg (indicated by the letter $X$ on Index Map 4).

Order address: Centre d'Etudes et de Réalisations Cartographiques Géographiques du CNRS, 191, rue Saint-Jacques, 75005, Paris, France; also available from BRGM.

CARTES GEOLOGIQUES DE LA MARGE CONTINENTALE
Géologie et structure à 1/250 000

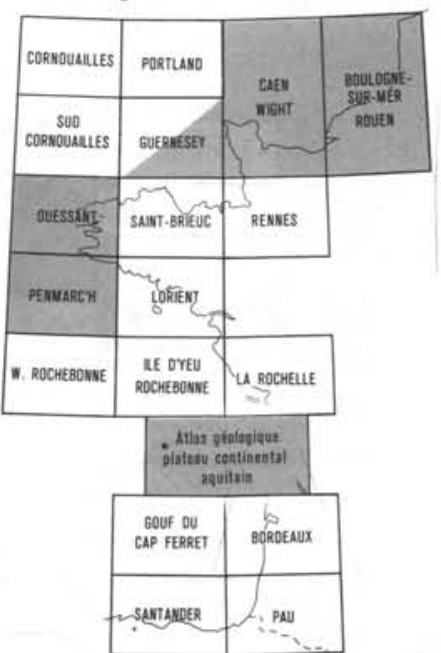

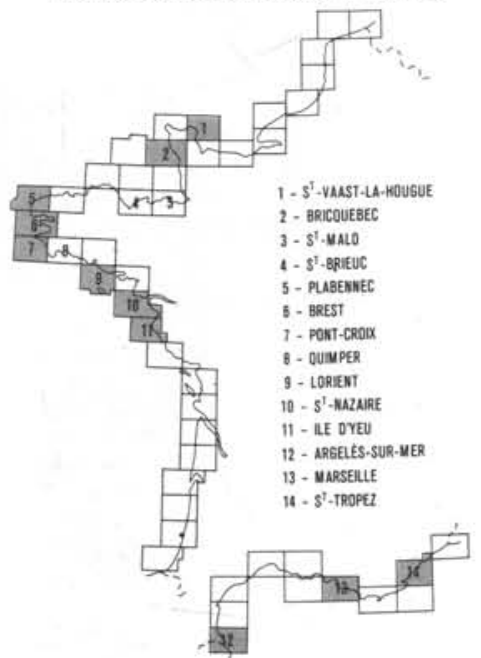

Nature des dépóts meubles à 1/100 000

Index Map 5: Carte géologique de la marge continentale, 1:100 000

Index Map 6: Carte géologique de la marge continentale, 1:250 000 


\section{Geophysics}

Carte gravimétrique de la France, géologie et anomalies de Bouguer - 1:320 000 - Orléans: BRGM (indicated by the letter G on Index Map 1).

Carte gravimétrique de la France - 1:200 000 - Orléans: BRGM. (See Index Map 7).

Carte gravimétrique de la France - 1:80 000 - Orléans: BRGM. (indicated by the letter G on Index Map 3).

Carte magnétique de la France - 1:250 000 - Orléans: BRGM. Sheets published: Marseille, Nice, Valence.

Carte magnétique de la France - 1:80 000 - Orléans: BRGM (indicated by the letter $M$ on Index Map 3).

Carte magnétique - 1:50 000 - Orléans: BRGM. Ozalid (indicated by the letter $\mathrm{M}$ on Index Map 4).

\section{Hydrogeology}

Carte hydrogéologique de la France - 1:50 000 - Orléans: BRGM., irregular (See Index Map 4).

Carte de vulnérabilité des eaux souterraines à la pollution 1:250 000 - Orléans: BRGM., irregular, Sheets published: Lyon, 1975; Valence, 1979.

Carte de vulnérabilité des eaux souterraines à la pollution 1:50 000 - Orléans: BRGM., irregular (See Index Map 4).

Miscellaneous

Carte des substances utiles - 1:50 000 - Orléans: BRGM., irregular (indicated by the letter S on Index Map 4).

Carte des zones exposées à des risques liés aux mouvements du sol et du sous-sol - var. scales 1:10 000, 1:20 000, 1:25 000 - Orléans: BRGM., irregular (indicated by the letter $Z$ on Index Map 4).

\section{ORDER ADDRESS FOR BRGM PUBLICATIONS:}

BRGM - Division Edition et Vente, B.P. 6009 ,

45060 ORLEANS CEDEX, France.

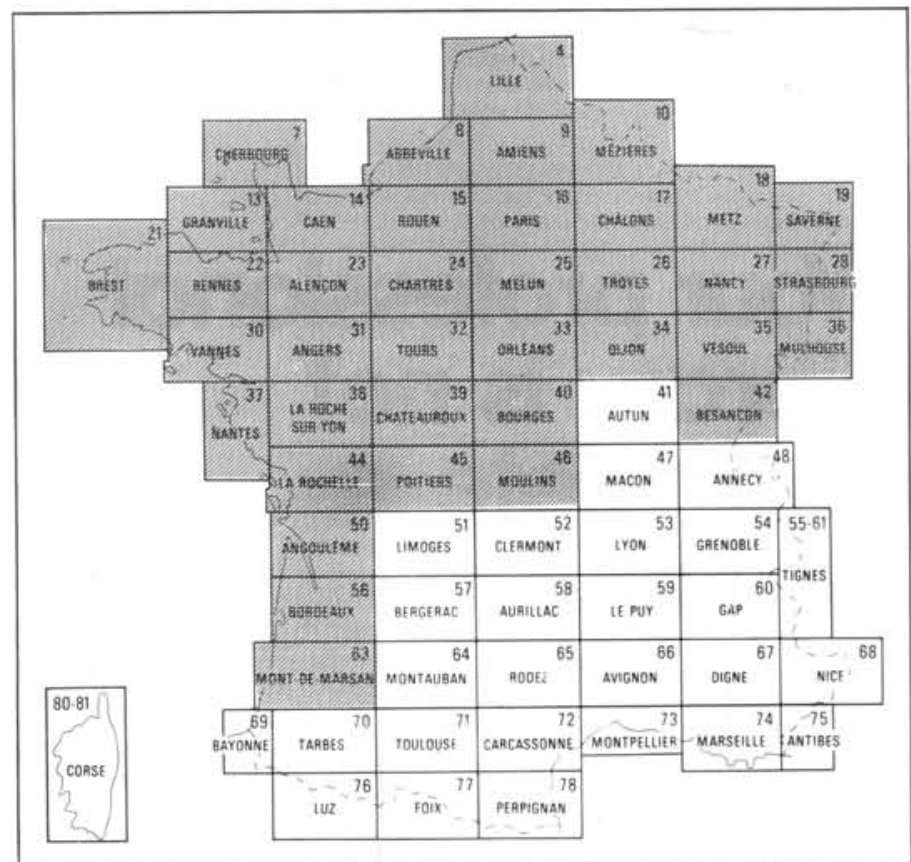

Index Map 7: Carte gravimétrique, 1:200 000

\section{IUGS PUBLICATIONS}

New IUGS Publication Series

\section{RECENT RELEASES}

- Sheng Shen-fu. THE ORDOVICIAN SYSTEM IN CHINA, Correlation Chart and Explanatory Notes. IUGS Publication No. 1, (1980), 7p., 3 figures, 6 tables and 1 correlation chart, $\$ 6.00$.

- Dean, W.T. THE ORDOVICIAN SYSTEM IN THE NEAR AND MIDDLE EAST, Correlation Chart and Explanatory Notes. IUGS Publication No. 2, (1980), 22p., 1 figure and 1 correlation chart, $\$ 6.00$.

- Webby, B.D. THE ORDOVICIAN SYSTEM IN AUSTRALIA, NEW ZEALAND AND ANTARCTICA, Correlation Chart and Explanatory Notes. IUGS Publication No.6, (1981), 64p., 1 table and 1 correlation chart, $\$ 6.00$

NB: Order all three for only $\$ 15.00$ total.

Please send me:

IUGS Publication No. 1. The Ordovician System in China.

- IUGS Publication No. 2. The Ordovician System in the Near and Middle East.

IUGS Publication No. 6. The Ordovician System in Australia, New Zealand and Antarctica.

Price: $\$ 6.00$ U.S. each or $\$ 15.00$ for all three.

My cheque for $\$$ is enclosed. Please bill me.

Name and Address:

Post this form to: EPISODES Secretariat, Room 177, 601 Booth Street, Ottawa, Conada, K1A OE8. 
